\title{
Pain on Injection: Propofol MCT Vs Propofol LCT
}

\author{
Dr. Sanjot S. Ninave and Dr. Bhavitha Venigalla* \\ Department of Anaesthesiology, Dattte Meghe Institute Medical Sciences, India
}

\begin{abstract}
Article Type: Article
Article Citation: Dr. Sanjot S.

Ninave and Dr. Bhavitha Venigalla. Pain on injection: propofol MCT vs propofol LCT. Indian Journal of Science and Technology. 2020; 13(02), 213-217. D0l: 10.17485/ijst/2020/ v013i02/149259
\end{abstract}

Received date: November 28, 2019

Accepted date: December 10, 2019

*Author for correspondence:

Dr. Bhavitha Venigalla

bhavitha123@gmail.com 9

Department of Anaesthesiology, Dattte

MEGHE Institute Medical Sciences, India

\section{Abstract}

Background: Propofol is one of the safest $d r y$ gr the induction of general anesthesia. The routinely availablo reparati $h$ in market being Propofol LCT (long chain trigly ctides, which do have a disadvantage of pain on injection. Th pai is ve, discomforting for the patient and also anesthe solos so th $-y$ are coming up with new preparation Propofo ICT (me iu, chain triglycerides) which do have a property of rea tion of pain on injection. In view of which we com arc the occ rence of pain on injection of Propofol MCT with ropofol " $\mathrm{CT}$ and rise in serum triglyceride levels after single indu tion do . Materials and methods: After obtaining instit al ethrommittee approval, 150 patients of ASA I \& II were $\epsilon$ rol"-ant the study undergoing various elective surgerie ar er ge eral anesthesia, where propofol is used as an induc on ac nt. Th study patients were divided into two groups wh rea ed Propofol MCT (Group M) and Propofol LCT (Group L) as inductio drug. The intensity of pain is evaluated by using visual ana vue score (VAS). Similarly, side effects of Propofol MCT were observal. Results: Group M showed reduced pain score $(4.15 \pm$ 1.90) after Propofol MCT injection along with less incidence of pain mpared to Group L $(6.37 \pm 2.49)$. Serum triglyceride levels had no significant difference in preoperative and postoperative values. Conclusion: In conclusion, pain on injection with Propofol MCT $(4.15 \pm 1.90)$ is less compared to Propofol LCT $(6.37 \pm 2.49)$; there is no evidence of elevation of triglyceride levels after single induction dose in the study.

Keywords: Propofol, Pain on Injection, Propofol MCT, Propofol LCT, Serum Triglycerides

\section{Introduction}

Propofol is a substituted Isopropylphenol that is administered intravenously as $1 \%$ solution in an aqueous solution of $10 \%$ soyabean oil, $2.25 \%$ glycerol, and $1.2 \%$ purified egg phosphatide [1].

Propofol is currently the preferred intravenous general anesthetic drug with a smooth induction, pleasant sleep, rapid recovery, and low incidence of nausea and vomiting. 
Despite these positive properties, it also has adverse effects such as injection pain, which may discomfort in the induction of anesthesia [2].

The mechanism of pain on injection of propofol is thought to be multifactorial but its exact causation is not clear. The most commonly identified mechanism is the release of bradykinin as a result of the activation of the plasma kinin-kallikrein system by propofol.

Lignocaine though being effective in reducing pain caused by propofol, there is a need for inflating tourniquet giving the drug intravenously, and then giving compression and deflating the tourniquet to overcome this disadvantage, there is a need of propofol solution which by itself is painless, so we want to compare the efficacy of Propofol MCT with that of routinely used Propofol LCT for the incidence of pain during inject

Regarding potential risks related to propofol, however, an increas n serum riglyceride levels has been described repeatedly, particularly after long-last g in vions, h critically ill patients presenting with deranged metabolic or enzymatic stem pro aged propofol administration might result in an excessive fat load wi e ang $\mathrm{p}$ Acreatitis, which is a well-known complication of hyper triglyceride [3]. 1 Acidence of serum triglygeridemia is also compared between both the grou $\mathrm{A}_{\mathrm{A}}$ checking preoperative and postoperative triglycerides.

\section{Aim}

To study the efficacy of Propofo' $\mathrm{MCT}$ ver $r$ tinely use Propopfol LCT in attenuating the pain caused by propofol injerion comparing serum triglyceride levels preoperatively and postoperatively.

\section{Materia and Methods}

The study ras ca ried ou in the Department of Anaesthesiology, JNMC, Sawangi (Meghe), Wardha. A Approval from the Institutional Ethics Committee,150 patients aged between 18-6. years willing to give written informed consent fitting into the inclusion criteria were included in this double blinded prospective observational study scheduled for various elective surgeries under general anesthesia. Patients are divided into groups into 75 each. Group MCT received 25\% of propofol MCT induction dose. Group LCT received $25 \%$ of propofol LCT. Preanesthetic evaluation was done a day before the surgery. Patients were asked to be nil by mouth by 8 hours. All the necessary routine investigations were noted along with preoperative triglycerides. Patients with chronic pain disorder, known allergy to the study drug, pregnancy, abnormal renal and liver function were excluded from the study. On arrival of patient to operation theater, all routine monitors are attached to the patient, and baseline parameters such as HR, SBP, DBP, MAP, SPO2 were recorded. Intra-venous cannulation was done using wide bore cannula (18 G or 20 G) on the dorsum of the hand or fore arm. Premedications were delebirately avoided to avoid influence on study results. With a tourniquet in place distal to venous cannulation, $2.5 \mathrm{ml}$ of total $10 \mathrm{ml}$ propofol, i.e., $25 \%$ of the induction dose, is given according to the 
groups divided. Patients are asked to indicate the severity of pain on injection using VAS Score at 25 seconds. This is the end point of the study and further procedure was carried out in conventional manner depending on the type of surgery. Awareness of the pain due to propofol after general anaesthesia is not considered. Postoperative serum triglyceride levels were measured.

\section{Statistical Analysis}

Assuming VAS score of Propofol MCT 2.089 and SD of 0.896, keeping at $80 \%$ and confidence interval at $95 \%$ (alpha error at 0.05 ), a sample of 60 patie ss would be required to detect a minimum of $25 \%$ of pain on IV Propofol MCT and LC 5 . nclude 75 patients in each group to compensate for possible drop out.

\section{Discussion}

Propofol is the most commonly used inducing a ent in the practice of anesthesia providing smooth induction, rapid recovery, and low inc ence of ausea and vomiting. Despite its positive effects, pain on propofol injection is rank 1 th mong the most important 33 low morbidity clinical anesthesia problems b, a po of expert anesthesiologists [4]. Table 1 shows patient characteristics pert $\mathrm{g}$ to emographic details and ASA grade did not differ between both the groups able show the VAS score in Propofol LCT $(6.37 \pm 2.49)$ is more compared to Propg $\mathrm{l}$ l (4.15 \pm 1.90). Similarly, there is less injection pain with Propofol MCT/LCT $=0.000,[5]$. Table 2 shows that incidence of pain is more in Propofol LCT (867\%) con ared to Propofol MCT (72.0\%). Propofol MCT/LCT had significantly lower ncidence of pain on injection in comparison with standard propofol group (37\% vs 65\% Tabl 2 compared the induction time in Group L (34.07 \pm 2.68$)$ and Group M (3 .01 2.7 ar a it was found to be similar. Table 3 Compared the preoperative and post erat atriglycrides which showed no significant difference between both the groups faile to increase triglyceride levels to a significant level; despite the difference in the lipid corent, single dose of MCT/LCT or LCT propofol did not increase serum triglyceride levels significantly to cause any adverse effects [7] which demonstrated that both LCT and MCT-LCT propofols cause significant rise in triglyceride levels in children when used for induction and maintenance of anesthesia. However, children in MCT-LCT

TABLE 1. Demographic data of the two groups - Propofol LCT and Propofol MCT

\begin{tabular}{llll}
\hline MAP & N & Group L & Group M \\
\hline $\begin{array}{l}\text { Age (mean } \pm \text { SD) } \\
\text { Gender }\end{array}$ & 75 & $44.31 \pm 11.29$ & $41.85 \pm 10.19$ \\
Male [n(\%)] & 75 & $40(53.3)$ & $41(54.7)$ \\
Female [n(\%)] & & $35(46.7)$ & $34(45.3)$ \\
Weight (mean \pm SD) & 75 & $59.67 \pm 9.53$ & $61.92 \pm 12.51$ \\
ASA Grade & & & \\
ASA I [n(\%)] & 75 & $57(76.0)$ & $59(78.7)$ \\
ASA II [n(\%)] & 75 & $18(24.0)$ & $16(22.3)$ \\
\hline
\end{tabular}


TABLE 2. Comparison of mean VAS pain score, induction time, and presence of pain between the two groups - Propofol LCT and Propofol MCT

\begin{tabular}{llllll}
\hline MAP & N & Group L & Group M & P-value & Sig. \\
\hline VAS pain score $($ mean \pm SD) & 75 & $6.37 \pm 2.49$ & $4.15 \pm 1.90$ & $<0.001$ & $\mathrm{~S}$ \\
Induction time in seconds (mean \pm SD) & 75 & $34.07 \pm 2.68$ & $33.61 \pm 2.72$ & 0.306 & NS \\
Pain present $[\mathrm{n}(\%)]$ & 75 & $65(86.7)$ & $54(72.0)$ & 0.027 & $\mathrm{~S}$ \\
\hline
\end{tabular}

P-value derived from independent sample t-test; P-value derived from chi-square test; significant at $\mathrm{p}<0.05$.

TABLE 3. Comparison of pre- and posttriglycerides values of group Pronofol LCT and Propofol MCT

\begin{tabular}{llll}
\hline \multirow{2}{*}{ Triglycerides } & $\mathbf{N}$ & Group L & Mean \pm S.D. \\
\cline { 2 - 4 } & & $128.94 \pm 21.6$ & 0.174 \\
Pre-op triglycerides & 75 & $134.41 \pm 2.62 \pm 21.59$ \\
Post-op triglycerides & 75 & 0.099 \\
P-value & & NS \\
Significance & &
\end{tabular}

group had lower triglyceride leve an a Idren in LCT group at the end of propofol infusion and 4 hours after tern natio [8] $\mathrm{m}$ ich stated that increased serum triglyceride level after propofol infusig is a ociated with increased risk of pancreatitis, coronary artery disease. It occure $\mathrm{CU}$ pat $\mathrm{nts}$ who receive long-term propofol infusion (>24 hrs) [9-10]. But this ropofor fusions are not now routinely used as newer and better drugs like dexme ctomi line and others have taken over propofol. No studies have cited that there was inc in se um triglycerides after single bolus dose of propofol except a case of 21 ar $\mathrm{d}$ pa ie operated for Bartholin duct excision developing pancreatitis after sing do conopofol [11]. It is hypothesized that concentration of free propofol in the aqueo phase of emulsion is responsible for the pain of injection. Various options were tried for the prevention of injection pain caused by propofol with varying degree of success, one among is the use of lidocaine. There is a sole need of propofol solution which by itself is painless or less painful. The drawback of propfol being pain on injection may be distressing to patient, interfere with smooth induction, which can be attenuated by a formulation in medium-chain triglycerides rather than long-chain triglycerides. Our study compared Propofol MCT and LCT for pain on injection instead of emulsions and found that Propofol MCT is better as compared to Propofol LCT.

\section{Conclusion}

Propofol MCT is associated with less incidence of pain on injection compared to Propofol LCT, as it also offers advantage without the addition of any other drugs. Also, the formulations did not increase serum triglyceride levels after single induction dose. 


\section{References}

1. Flood P, Rathmell JP, Shafer S. Stoelting's pharmacology and physiology in anaesthetic practice. $5^{\text {th }}$ edn. $7^{\text {th }}$ Indian reprint. 2018, 160. DOI: 10.1097/ALN.0000000000000646

2. Beyaz SG, Eman A. Injection pain of propofol in children: A comparison of two formulations without added lidocaine. Journal of Anaesthesiology Clinical Pharmacology. 2012; 28(3), 314317. DOI: $10.4103 / 0970-9185.98322$

3. Hermann J. Theilen, Sigrid Adam, Albrecht MD, Raga M. Propofol in a medium- and longchain triglyceride emulsion: pharmacological characteristics and potential beneficial effects. Anesthesia \& Analgesia. 2002; 95(4), 382-384. DOI: 10.1213/00000539-20 000024

4. Macario A, Weinger M, Truong P, Lee M. Which clinical anesthesia out mes are th common and important to avoid? The perspective of a panel of expert anae hes ogists. nesthesia \& Analgesia. 1999; 89, 652-658. DOI: 10.1097/00000539-1999050/ -00023

5. Rau J, Roizen MF, Doenicke AW, O'Connor MF, Strohschneide U. opofol in an emulsion of long- and medium-chain triglycerides: the effect on pain anesthes \& algesia. 2001; 93(2), 382-384. DOI: 10.1097/00000539-200108000-00029

6. Larsen B, Beerhalter U, Biedler A, Brandt A, Doeg Prün K, A dkönig R, Larsen R. Less pain on injection by a new formulation of propofol? comparison with propofol LCT. Anaesthesist. 2001; 50(11), 842-845. DOI: 10.1007/s00101-00 0234-0

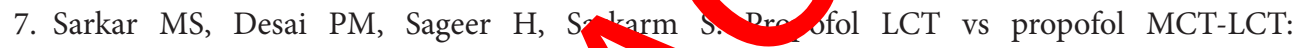
randomized controlled trial. Indian Journ loj mical Anaesthesia. 2016; 3(2), 214-218. DOI: 10.5958/2394-4994.2016.00039.1

8. Bhukal I, Thimmarayan G, Bal , Sola ki SL, Samra T. Comparison of serum triglyceride levels with propofol in long chair tre vo chae and propofol in medium and long chain triglyceride after short term anesthe in pedic ic patients. Saudi Journal of Anaesthesia. 2014; 8(5), 53-56. DOI: $10.4103 / 1658-334$ X.1 1076

9. Devlin JW, Lau A , Tanios M. Propofol-associated Hypertriglyceridemia and pancreatitis in the intensive e uni analysis of frequency and risk factors. Pharmacotherapy. 2005; 25(10), 1348-1352 DOI. 1592 Mco.2005.25.10.1348

10. Sundary niti I Boon $\mathrm{N}$, Chalacheewa T, Jommaroeng P, Rungsithiwan W. A comparison of propo L LC - 2 ropofol-LCT/MCT on pain of injection. Journal of the Medical Association of Thailan 2007; 90(12), 2683-2688. https://www.ncbi.nlm.nih.gov/pubmed/18386721

11. Jawaid Q, Proti ME, Neuschwander-Tetri BA, Burton FR. Acute pancreatitis after single-dose exposure to propofol: a case report and review of literature. Digestive Diseases and Sciences. 2002;47:614-618. DOI: 10.1023/a:1017932522875 